\title{
Food related asthma: a difference between two ethnic groups
}

\author{
N M WILSON \\ Department of Paediatrics and Neonatal Medicine, Royal Postgraduate Medical School, \\ Hammersmith Hospital, London
}

SUMMARY A survey of reported, food related asthma was carried out among children referred to hospital. Ninety nine replies were received from a postal questionnaire and 78 other children were interviewed personally. Both survey methods gave similar results. Symptoms from at least one item of diet were significantly more frequently reported by Asian than non-Asian children (91\%:58\% respectively). Ice, fizzy drinks, fried food, and nuts were incriminated significantly more often by the Asian children, but there was no difference in the prevalence of asthma associated with orange squash, milk, chocolate, or eggs. This ethnic difference could not be explained by a difference in severity of asthma. Results are presented of six Asian children who showed increased bronchial responsiveness after eating chips but not potato, thereby confirming their claim of asthma induced by fried foods. This report shows that food as a trigger of asthma is much more common than is generally accepted, particularly in Asian children.

There are very few data on the prevalence of food or drink induced symptoms in asthmatic children, as even a strong history of asthma resulting from an adverse reaction to a particular food is only infrequently confirmed by double blind challenge. ${ }^{1}$ Apart from the obvious fallibility of any clinical history, there are many reasons why an asthmatic response to food may not be confirmed in the laboratory. ${ }^{2}$ The response may only be intermittent, possibly depending upon the severity of asthma (particularly variable in childhood) as well as the frequency and amount of exposure to the substance. Also, a response with a delayed onset is difficult to detect in the laboratory. In addition, the measurement of resting lung function alone may fail to detect an adverse response which can be shown by a test of bronchial responsiveness. ${ }^{3-5}$ Patients recognise the induced increase in bronchial responsiveness as an increased response to the various trigger factors of asthma such as exercise and laughter. So a history of food or drink induced asthma may be a more reliable guide than has previously been accepted.

This paper reports the results of a survey of food and drink induced symptoms in a group of hospital based asthmatic children. In view of the observation that children of Asian origin (families originating from India, Pakistan, or Sri Lanka) had been shown to develop increased bronchial responsiveness after challenge with ice ${ }^{4}$ and 'cola' drinks, ${ }^{5}$ the results obtained from Asian children have been compared with those obtained from children of other ethnic origins (non-Asians).

\section{Methods}

The survey consisted of two parts, a postal questionnaire and a personal interview.

Postal questionnaire. A postal questionnaire was sent to 75 Asian children, aged between 3 and 17 years, and 75 age and sex matched non-Asian children. Both groups were randomly selected from the files of the asthma clinic. The children or their parents were asked to record any increase in cough or wheeze considered to result from ingestion of any substance from a suggested list (Table 1) or from any other item of their diet. A totally negative reply consisted of one that recorded no induced asthma from any substance. Encouragement was given to return the questionnaire in a prepaid envelope, even if the answers were all negative.

Personal interview. Seventy eight consecutive children, not included in the postal survey, needing daily treatment for perennial asthma and attending 
Table 1 Details of patients and prevalence of food induced symptoms

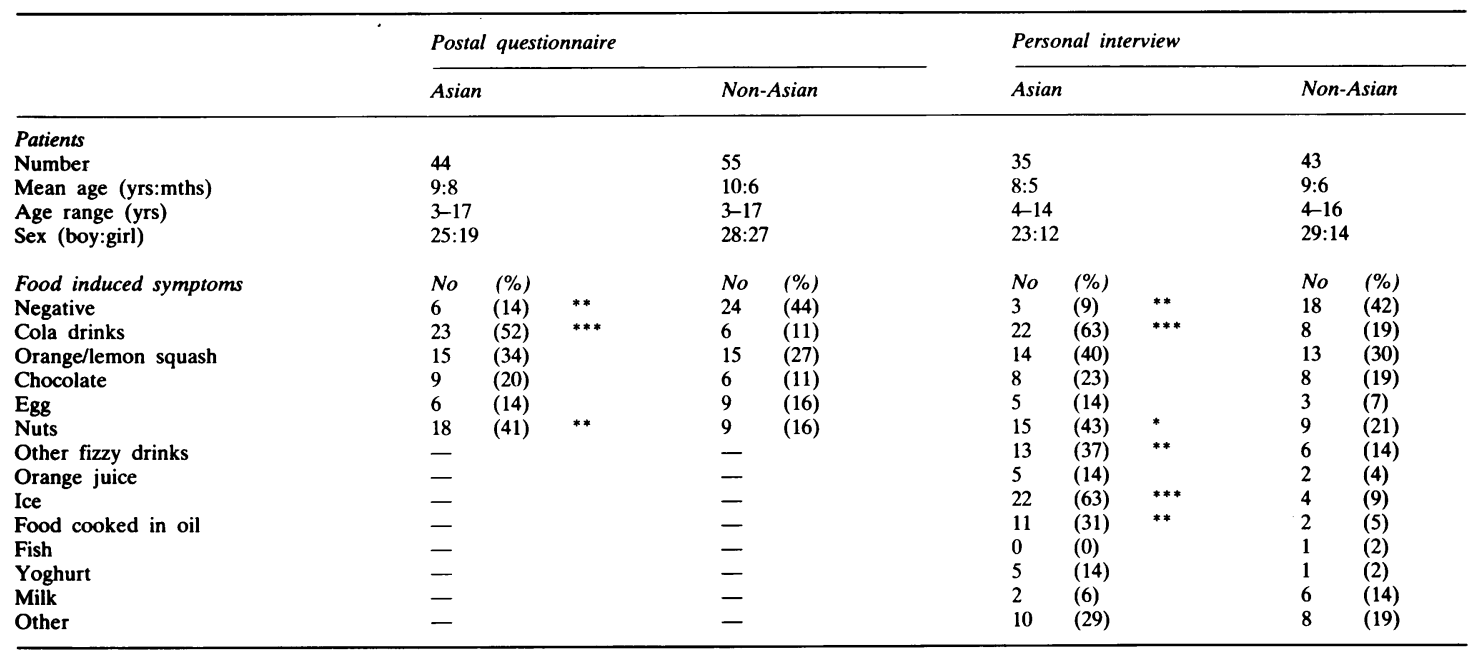

${ }^{* * *} \mathrm{P}<0 \cdot 001 ;{ }^{* *} \mathrm{P}<0 \cdot 001 ;{ }^{*} \mathrm{P}<0.05$.

one of two hospital asthma clinics were interviewed personally by the author. The children and parents were asked whether they considered cough or wheeze were induced by any of the specific items from a standard questionnaire (Table 1). An answer was only considered positive if symptoms were, at any time, severe enough to necessitate additional bronchodilator treatment. A tickling sensation in the throat occurring while eating and which induced coughing was ignored. The interval between ingestion and time of onset of symptoms was also recorded. A note was made of food associated symptoms other than asthma, but for the purpose of this study they are not included in the results.

The significance of differences between the two ethnic groups and between the two survey methods were assessed by $\chi^{2}$ analysis using the Yates's correction or non-paired Student's $t$ test, as appropriate.

\section{Results}

Postal survey. Replies to the postal survey were received from 99 of $150(66 \%)$ patients sent a questionnaire. The reply rate was lower for Asians (44 of $75(59 \%)$ than non-Asians (55 of $75(73 \%)$, but the difference was not statistically significant. Because of the low rate of response, an overall prevalence of food related symptoms could not be calculated. Certain previously unsuspected differences between ethnic groups became evident. The Asian children reported significantly more symptoms from 'cola' drinks $(\mathrm{P}<0.001)$ and nuts
$(P<0 \cdot 01)$ (Table 1) than the non-Asian children. There was no difference between the groups for orange squash, chocolate, or eggs.

Personal questionnaire. Thirty five Asian and 43 non-Asian (one West Indian, two Chinese, and the rest Caucasian) children were interviewed personally. For the subjects as a whole, $73 \%$ considered at least one of the listed items caused symptoms, but a positive history was significantly more frequently obtained from the Asian group $(\mathrm{P}<0 \cdot 01$; Table 1$)$. This was due to a significantly higher prevalence of symptoms induced by 'cola' drinks $(\mathrm{P}<0.001)$, ice cold drinks $(P<0.001)$, nuts $(P<0.05)$, and food fried in oil $(P<0 \cdot 01)$. There was no significant difference between the two groups for orange squash, eggs, chocolate, or milk.

There was no significant age or sex difference between the two ethnic groups, although the Asian children tended to be younger and to use less prophylactic medication (Tables 1 and 2). When only those with a positive history from both ethnic groups were compared the mean age of the Asian children was significantly lower, and they had less severe asthma (as judged by their current medication) than the non-Asian children (Table 2). There was, however, no significant difference in age, sex distribution, or severity of asthma, between nonAsian children with and without a history of food related symptoms.

The time of onset of symptoms after ingestion varied between a few minutes and 24 hours. Almost $50 \%$ of the children considered that their symptoms 
Table 2 Characteristics of children personally interviewed with and without a positive history

\begin{tabular}{|c|c|c|c|c|}
\hline & \multicolumn{2}{|l|}{ Asian } & \multicolumn{2}{|l|}{ Non-Asian } \\
\hline & Positive & Negative & Positive & Negative \\
\hline Number & 32 & 3 & 25 & 18 \\
\hline Age $(\mathrm{yrs})$, mean $(\mathrm{SD})$ & $8 \cdot 5 \longdiv { ( 2 \cdot 8 ) }$ & $8 \cdot 8(3 \cdot 8)$ & $10 \cdot 4(3 \cdot 16)$ & $8.5(3.38)$ \\
\hline Sex, boy:girl & 21:11 & $2: 1$ & $16: 9$ & $13: 5$ \\
\hline \multicolumn{5}{|l|}{ Asthma treatment (\%) } \\
\hline$\beta$ agonist alone & 28 & 33 & 0 & 11 \\
\hline Theophylline/cromoglycate & 25 & 33 & 48 & 44 \\
\hline Steroids:inhaled/oral & 47 & 33 & 52 & 44 \\
\hline
\end{tabular}

${ }^{* *} \mathrm{P}<0.02$.

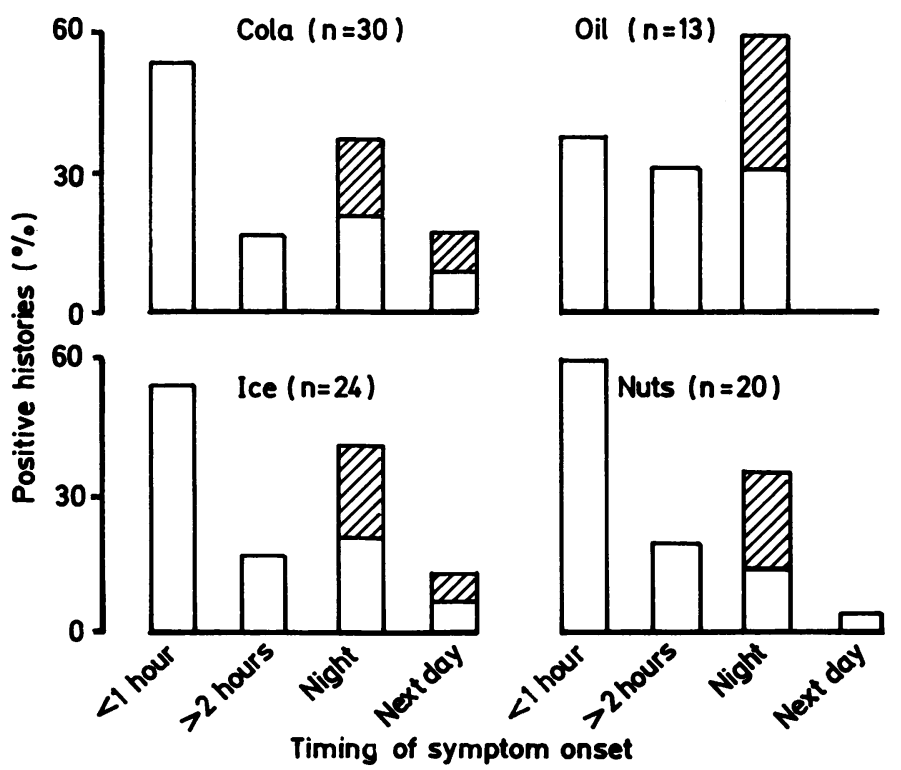

Fig. 1 Timing of onset of symptoms after ingestion (open columns) and return of symptoms (hatched columns) in those children with a history of cough or wheeze after ice, cola, nuts, and food cooked in oil.

Night=nocturnal symptoms irrespective of time of ingestion.

recurred, or were first noticed, during the following night. The timing of the onset of symptoms was similar for classic allergens such as milk, egg, and nuts and for physical or chemical stimuli such as ice or cola drinks, and did not differ between the two ethnic groups (Fig. 1).

There was no significant difference in the answers obtained by the two survey methods, where the same question was asked. The ethnic difference in the reporting of food and drink induced asthma was the same for both methods.

\section{Discussion}

Both methods of survey found a surprisingly high number of children incriminating at least one of the suggested items. Although many patients in the postal survey failed to return the questionnaire the relative reply rate between Asian and non-Asian children was not statistically different, so the ethnic difference is unlikely to be explained by a lower return rate among Asians with a negative questionnaire. The fact that personal interviews and a postal survey gave similar results suggests that the results were not merely due to unrepresentative sampling.

There are no comparable studies of food related cough and wheeze in asthmatic children. Anderson et al $^{6}$ stated that only $6 \%$ of a group of 8 to 10 year olds gave food as a precipitating factor in their asthma. That study, however, was dealing with the general population of asthmatic children rather than those referred to hospital. Even if food had been a precipitating factor, with milder symptoms of asthma the association would have been more difficult to make. In addition, a general question, as used in their survey, would be expected to give a 
lower positive reply rate than a specific list. In the present study, $25 \%$ of those children who subsequently incriminated specific food items had given a negative reply to a preliminary general question.

There are no previously published data on ethnic differences in food sensitivity. Several substances such as orange squash, chocolate, milk, and eggs were similarly incriminated by the two groups. The increase in a history of food and drink related asthma among the Asian children depended on significantly increased incrimination of specific items, namely 'cola' drinks, nuts, food cooked in oil, and ice (Table 1). Until recently asthma induced by 'cola' drinks and ice had not been reported but these claims have now been confirmed in the Asian subjects tested, by showing increased bronchial responsiveness to nebulised histamine in placebo controlled food challenge tests. ${ }^{34}$ Using the same protocol, six Asian children who gave a history of asthma exacerbated by fried food were challenged with chips and the same volume of baked potato on separate days in a single blind fashion. The response was monitored by serial peak expiratory flow measurements and the sensitivity to inhaled histamine (the concentration of histamine that induced a $20 \%$ fall in peak expiratory flow) was measured before and after food ingestion. The latter test was used as a measure of bronchial responsiveness. The results (Fig. 2) show that ingestion of oil (but not potato) could indeed induce symptoms of asthma by increasing bronchial responsiveness (shown by a fall in the amount of histamine that induced a $20 \%$ fall in peak expiratory flow). There was no associated change in baseline peak expiratory flow, so without the histamine test the response would not have been detected by the normal laboratory challenge procedure. This adverse response to fried food has not previously been reported but confirms the claims of all six Asian children tested.

The confirmation of a history of asthma exacerbated by ice, ${ }^{4}$ 'cola' drinks, ${ }^{3}$ and oil excludes the possibility that these substances were incriminated by Asian children due to cultural folklore. The reason for this ethnic difference in adverse reactions to food and drink, however, remains unexplained. One possibility is that the non-Asian children were less aware of food related symptoms, but this seems unlikely as several items were similarly incriminated by both groups. Neither can the difference be explained by a greater severity of asthma in the Asian children as they used less medication than the non-Asians. The younger age of the Asian children is not likely to be the explanation either as nonAsian children with a positive history tended to be older than those without (Table 2). A genetic or dietary difference may be a possibility. Interest-
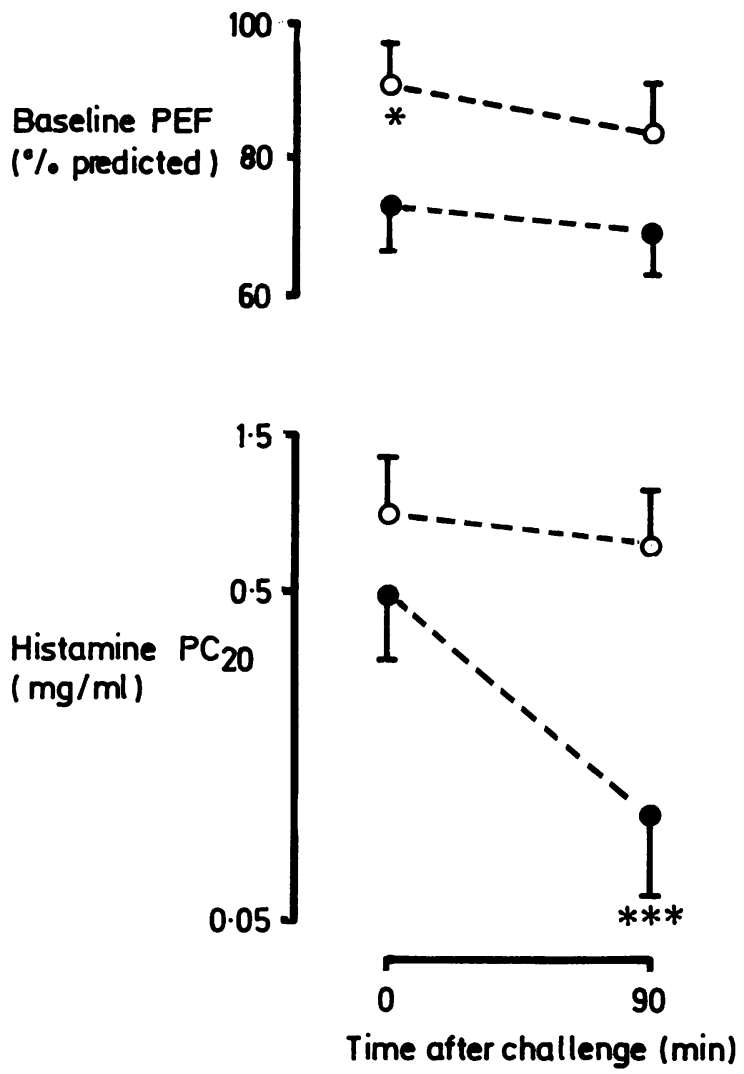

Fig. 2 Baseline peak expiratory flow (PEF) and amount of histamine that induced a $20 \%$ fall in PEF (histamine $P C_{20}$ ) before and after challenge with chips (oil) and baked potato (placebo) $\bigcirc$.

${ }^{* * *} \mathrm{P}<0 \cdot 001 ;{ }^{*} \mathrm{P}<0 \cdot 05$.

ingly, the pattern of food and drink induced asthma reported by 59 children attending an asthma clinic in Colombo, Sri Lanka, was very similar to that of our Asian children. Fifty three per cent of those questioned incriminated ice, $36 \%$ coloured drinks, and $19 \%$ fried foods. The only difference was that in Sri Lanka, citrus fruits and coconut were more frequently related to symptoms of asthma and the consumption of fizzy drinks was negligible ( $\mathrm{N}$ de Sylva, personal communication).

Morrison-Smith and co-workers ${ }^{7}$ reported that asthma was less common among Asian immigrant children than the general population, but in Birmingham it has recently been noted that Asian asthmatics were relatively more frequently admitted to hospital with acute attacks (J G Ayres, personal communication). There are social reasons that may 
account for this higher admission rate among Asians, but adverse reactions to food could be a factor.

The confidence placed in a survey of food induced asthma which is based on history alone depends upon the validity of the history. May ${ }^{1}$ was only able to confirm the history of an asthmatic response to food in 11 of 38 children tested. Similar experience by workers has resulted in a mistrust of reports of food associated symptoms. The results, however, of this study (shown in Fig. 2) and those previously reported which use a histamine test to detect food induced asthma,${ }^{2-5}$ show that it is rarely possible to detect the adverse airway reaction by measuring serial peak expiratory flow alone. The resulting increased bronchial responsiveness is only infrequently associated with changes in resting baseline peak expiratory flow or of more sensitive tests of airways function, such as the specific airways conductance (personal observation). An additional trigger is needed to show the increased bronchial responsiveness. These laboratory findings were substantiated by the children themselves during questioning. They often stated that a reaction to a particular food could be expressed as an increased susceptibility to other trigger factors of asthma, such as exercise and laughter. In some, the reaction to food often occurred at night (Fig. 1), at a time when bronchial responsiveness is known to be increased. ${ }^{8}$ Also, patients reported that adverse reactions to foods depended on the current severity of their asthma, and so might only be present during a viral infection or in the pollen season. These observations could account for reported failure to confirm a history of food induced asthma by double blind challenge tests, performed in a laboratory, devoid of extraneous stimuli, and when the child is well. Because of the multifactorial nature of asthma, it cannot be assumed that a negative laboratory test means that a response never occurs. A definitive history is likely to have a basis in fact; a negative one could possibly mean failure of association.

Both from the evidence of histories and the challenge tests performed, a wide variety of ingested stimuli, including ice and chemicals, may induce an increase in bronchial responsiveness of a similar pattern. The underlying mechanism is far from clear and cannot be explained by a simple vagal reflex because of the timing of the changes, which are greater 90 minutes after challenge than at 30 minutes. ${ }^{4}$ As the pathophysiology of increased bronchial responsiveness in general is still controversial, further work on the mechanisms of food induced increased bronchial responsiveness, using specific blockers, may help to shed light on the pathogenesis of asthma.

The practical conclusion of this survey is the recognition that food induced symptoms in children with asthma are more common than is often recognised. Popular drinks such as orange and lemon squash are frequent offenders and Asian children are particularly susceptible to ingestion of ice, cola, and other fizzy drinks, as well as fried food and nuts. In most of the susceptible children, it seems likely that food sensitivity manifests itself as an increase in day to day symptoms of asthma but it is also worth remembering that it may present as an increase in nocturnal symptoms. Because of the suggestion, however, that an asthmatic response to ingested substances is more likely when the airways are most responsive, an important but unproved possibility is that food and drink sensitivity could be a factor in acute severe attacks of asthma.

I thank Dr Michael Silverman for his advice, Caroline Dixon and Helen Vickers for technical assistance, and Christine Wrage for typing the manuscript. Financial support was gratefully received from Boehringer Ingelheim (UK) Limited and the Asthma Research Council.

\section{References \\ 1 May CD. Objective clinical and laboratory studies of immediate hypersensitivity reactions to food in asthmatic children. J Allergy Clin Immunol 1976;58:500-15. \\ ${ }^{2}$ Wilson NM, Silverman M. The diagnosis of food sensitvity in childhood asthma. $J R$ Soc Med 1985;78(Suppl 5):11-15. \\ 3 Wilson N, Vickers H, Taylor G, Silverman M. Objective test for food sensitivity in asthmatic children: increased bronchial reactivity after cola drinks. $\mathrm{Br}$ Med $J$ 1982;284:1226-8. \\ 4 Wilson N, Dixon C, Silverman M. Increased bronchial respon- siveness caused by ingestion of ice. Eur J Respir Dis 1985;66: 25-30. \\ ${ }^{5}$ Hariparsad D, Wilson N, Dixon C, Silverman M. Oral tartrazine challenge in childhood asthma: effect on bronchial reactivity. Clin Allergy 1984;14:81-5. \\ - Anderson HR, Palmer JC, Brailey P, Cooper J, West S. A community survey of asthma and wheezing illness in 8-10 year old children. Report to SW Thames Regional Health Authority, 1981. \\ ${ }^{7}$ Morrison-Smith J, Harding LK, Cumming G. The changing prevalence of asthma in school children. Clin Allergy 1971;1: $57-61$. \\ ${ }^{8}$ deVries K, Goch JT, Bony-Noord H, Orie NGM. Changes during 24 hours in the lung function and hyperreactivity of the bronchial tree in asthmatics and bronchitics. International Archives of Allergy 1962;20:93-101.}

Correspondence to Dr N M Wilson, Department of Paediatrics and Neonatal Medicine, Hammersmith Hospital, Du Cane Road, London W12 0HS.

Received 19 April 1985 